\title{
Evolution des principes de la commutation assistée dans les onduleurs de tension. Présentation d'un onduleur haute fréquence à « commutations douces »
}

\author{
F. Forest et P. Lienart \\ Laboratoire d'électrotechnique, signaux et robotique, E.N.S. de Cachan, 61, avenue du président Wilson, \\ 94230 Cachan, France
}

(Reçu le 26 juin 1988, révisé le 10 octobre 1988, accepté le 17 octobre 1988)

\begin{abstract}
Résumé. - Cet article a pour objet l'étude d'un onduleur dont la structure est basée sur l'utilisation d'interrupteurs dit « résonnants ». Après avoir rappelé les différents modes de commutations utilisés au sein des onduleurs de tension et souligné les similitudes structurelles des différentes solutions, nous proposons un convertisseur dont les performances autorisent un fonctionnement en modulation de largeur d'impulsion à haute fréquence. Le choix d'un mode de commande particulier permet d'en exploiter totalement les caractéristiques. L'expérimentation d'un onduleur de $4 \mathrm{~kW}$, utilisant des transistors à grille isolée (I.G.B.T.) met en évidence les qualités de l'ensemble, tant sur le plan des performances dynamiques que du rendement.

Abstract. - This paper deals with a study about an inverter whose structure use the « resonant switch » concept. After having reminded the different switching modes commonly used among the voltage inverters, we suggest a converter whose performances provide a high frequency rate pulse width modulation operation. The choice of a particular control mode allows to take advantage of the features. The tests results of a $4 \mathrm{~kW}$ inverter using insulated gate bipolar transistors display the real qualities of the structure, particulary about dynamic performances and efficiency.
\end{abstract}

\section{Introduction.}

Dans le développement de la conversion d'énergie statique, nourri par l'apparition puis l'amélioration des composants de puissance à semi-conducteurs, les onduleurs de tension tiennent une place importante. Utilisés pour générer des systèmes de tensions alternatives, ils sont devenus un maillon indispensable dans la constitution d'entraînements à vitesse variable à l'aide de machines alternatives.

Ces onduleurs demeurent parmi les convertisseurs les plus délicats à mettre en œuvre du fait de leur structure particulière. La nécessité, dans de nombreuses applications, d'utiliser des fréquences de commande très supérieures à la fréquence fondamentale du signal alternatif généré, amplifie ces problèmes de mise en œuvre.

Si l'on observe l'évolution de la constitution de ces convertisseurs depuis l'apparition des premiers dispositifs de puissance commandés à semi-conducteur, les thyristors, on note que, si les modes de commutations des interrupteurs ont évolué, l'on retrouve constamment la nécessité d'assister totalement (thyristors, thyristors duaux) ou partiellement (composants entièrement commandés) l'une ou l'autre des commutations. Ceci s'accompagne invariablement de la présence autour du composant proprement dit, d'un certain nombre d'éléments additionnels. Ces éléments, volumineux dans les circuits d'extinction des thyristors, ont vu leur encombrement se réduire considérablement avec l'apparition des composants entièrement commandés.

Néanmoins, dans ce dernier cas, lorsque l'on veut utiliser des fréquences de commande supérieures à $10 \mathrm{kHz}$, on se heurte rapidement aux problèmes de commutation propres à la structure de l'onduleur de tension; la constitution des réseaux d'assistance devient rapidement inextricable sans pour autant apporter de solutions véritablement satisfaisantes aux problèmes sus-cités.

Une solution consiste à utiliser les propriétés des composants entièrement commandés dans des structures s'apparentant aux structures à thyristors, en éliminant les limitations dues aux performances 
dynamiques de ces derniers. Il est alors possible d'obtenir des commutations dites « douces », propres à faciliter le découpage à fréquence élevée.

Dans cet article, après avoir rappelé les différentes étapes de l'évolution des onduleurs de tension et souligné leurs similitudes de forme, nous proposons une structure d'onduleur de tension à commutations douces, adaptée à des fonctionnements au-delà de $20 \mathrm{kHz}$, et dont les performances sont totalement exploitées du fait de l'utilisation de modes de commande originaux. La réalisation d'un module 300 V-20 A, associée à l'utilisation d'I.G.B.T. (insulated gate bipolar transistor), composants réalisant une synthèse des caractères les plus intéressants des transistors MOS et bipolaire et parfaitement adaptés au mode de commutation choisi, confirme la validité des principes retenus.

\section{Structure de base de l'onduleur de tension.}

Cette structure de base est représentée figure 1. Elle résulte de l'association de deux interrupteurs, constitués d'un composant commandé et d'une diode antiparallèle. Une source continue, $E$, supposée parfaite, alimente l'ensemble. On s'attachera ici, comme dans la suite de l'exposé, à un fonctionnement correspondant à une commande de type « modulation de largeur d'impulsion». On suppose donc que le courant de sortie est constant pendant les commutations.

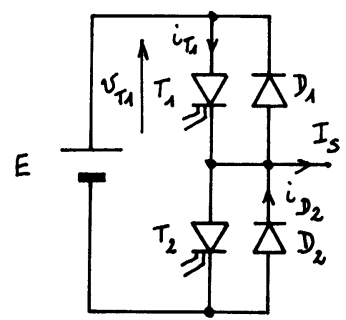

Fig. 1. - Convertisseur de base.

[Basic converter.]

Le composant élémentaire peut être, intrinsèquement, entièrement commandé (transistor bipolaire ou MOS, G.T.O., I.G.B.T.), ou réalisé à l'aide d'un thyristor, muni d'un circuit d'extinction contrôlable (thyristor auxiliaire).

Le comportement à l'ouverture est sensiblement différent selon que le composant est commandable ou non. Les deux configurations ont leurs avantages et inconvénients que nous allons maintenant préciser.

\section{Commutations dans les onduleurs à Thyristors.}

Le thyristor impose dans de telles structures l'introduction de circuits d'extinction. Un exemple très classique d'onduleur à thyristors, la structure de Mac-Murray [1], est donné figure 2a.

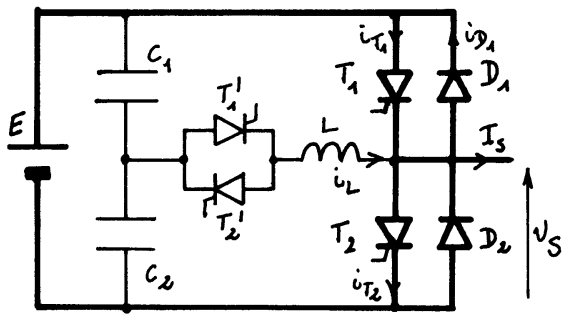

a)

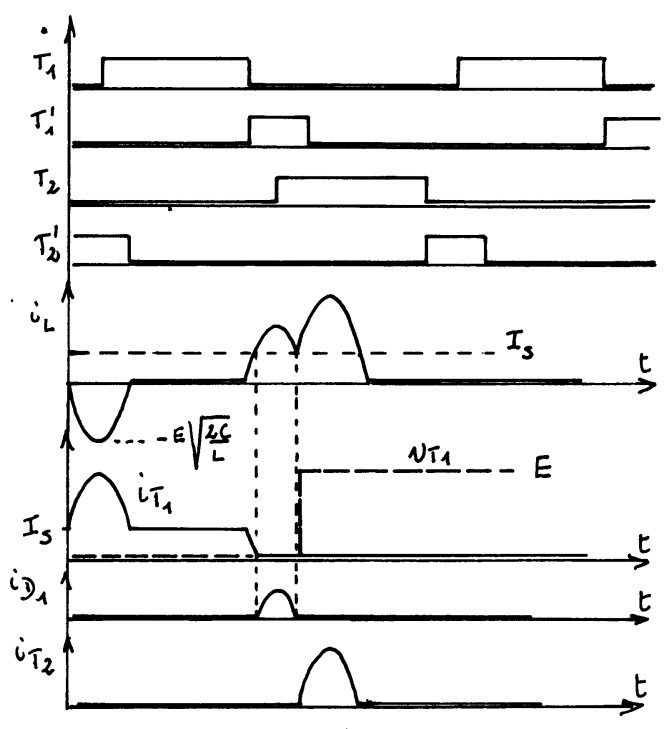

b)

Fig. 2. - Onduleur de Mac-Murray.

[Mac-Murray inverter.]

La séquence de commande et les principales grandeurs de commutation apparaissent figure $2 b$. Par principe, ces commutations assistées ne sont pas génératrices de pertes. La fermeture, commutation véritablement forcée, peut être assistée à l'aide d'une simple inductance (Fig. 3).

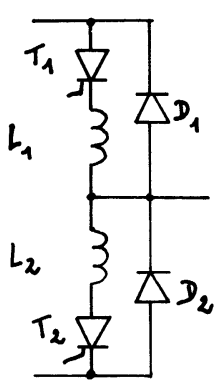

Fig. 3. - Inductances d'aide à la commutation.

[Switching aid inductances.] 
En pratique, les caractéristiques de thyristors font que ces circuits sont très volumineux et que les performances d'ensemble de l'onduleur sur le plan fréquentiel sont modestes $(f<2 \mathrm{kHz})$. De surcroît, ces circuits d'extinction imposent un surdimensionnement des composants, conséquence des surintensités qu'ils génèrent (Fig. $2 b$ ).

Enfin, ces circuits ayant un pouvoir de coupure limité, un raté de blocage, dû à une surcharge, conduira immanquablement à la mise en court-circuit du bras.

Il n'en reste pas moins que la nature de la commutation à l'ouverture est extrêmement intéressante car elle est non dissipative et permet l'introduction d'un réseau d'assistance à la fermeture n'induisant pas de pertes.

\section{Commutations dans les onduleurs à interrupteurs entièrement commandés.}

L'apparition des composants à semi-conducteurs entièrement commandés (transistors bipolaires et MOS, thyristors blocables, I.G.B.T.) a permis, dans un premier temps, de simplifier considérablement les convertisseurs, en éliminant les circuits d'extinction. Cette simplification est effective si les fréquences de commutation restent proches de celles des équipements à thyristors $(f<5 \mathrm{kHz})$ ou si les puissances mises en jeu restent faibles $(P<$ qqes $\mathrm{kW})$. $\mathrm{Si}$, dans des gammes de puissance plus élevées, on désire utiliser des fréquences de commutation supérieures à $10 \mathrm{kHz}$, les problèmes de commutation propres à la structure en pont, et qui n'ont pas d'influence notable en basse fréquence, prennent une importance considérable.

Ils ne diffèrent pas fondamentalement de ceux rencontrés dans des structures plus simples, tel le hacheur série, mais s'en distinguent car les solutions que l'on peut $y$ apporter sont complexes et insatisfaisantes.

Rappelons brièvement, pour illustrer ce propos, la nature des commutations, dans les structures en pont de cette nature.

Si l'on reprend le schéma de la figure 1, la nature des commutations résulte de l'association d'un composant commandé et d'une diode, et de la vitesse d'évolution du courant, imposée par ce composant (Fig. 4).

De ce fonctionnement résultent des pertes par commutation [6] :

- à l'amorçage

$$
P_{\mathrm{a}}=\frac{1}{2} E I_{\mathrm{s}} t_{\mathrm{m}_{i}} f+E Q_{\mathrm{r}} f
$$

$t_{\mathrm{m}_{i}}$ temps de montée de $i_{\mathrm{T} 1}$

$Q_{\mathrm{r}}$ charge recouvrée dans la diode

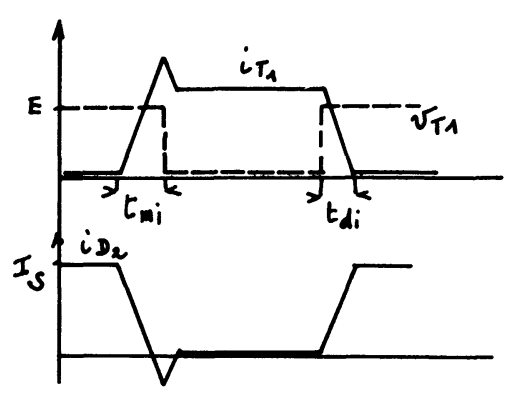

Fig. 4. - Mécanisme de la commutation forcée.

[Forced switching mechanism.]

- au blocage

$$
P_{\mathrm{b}}=\frac{1}{2} E I_{\mathrm{s}} t_{\mathrm{d}_{i}} f
$$

$t_{\mathrm{d}_{i}}$ temps de descente de $i_{\mathrm{T} 1}$

Augmenter les vitesses de commutation du composant n'apporte pas de solution satisfaisante à ce problème car, parallèlement, l'augmentation correspondante des contraintes en $\frac{\mathrm{d} i}{\mathrm{~d} t}$ et $\frac{\mathrm{d} v}{\mathrm{~d} t}$ provoque des effets secondaires tout aussi pénalisants (augmentation des pertes par recouvrement, perturbations sur les commandes).

On comprend, dans ces conditions, que la mise en cuvre d'un tel pont dans sa configuration élémentaire, à fréquence et puissance élevées, est délicate voire impossible.

Une démarche possible consiste alors à « greffer » sur les interrupteurs, des circuits propres à aider leurs commutations. Un exemple de réseaux [5, 6] est donné figure 5 .

De par leur association dans la structure en pont, ces réseaux ont un comportement caricatural (fortes pertes, contraintes élevées sur les composants qu'ils doivent assister). Il est possible d'améliorer quelque peu ces circuits en substituant aux diodes $D_{1}^{\prime}$ et $\mathrm{D}_{2}^{\prime}$, des composants commandés (Fig. 6) permettant de contrôler la phase de charge des condensateurs.

Ces interrupteurs sont fermés uniquement pendant l'ouverture du composant correspondant. La charge parasite est alors amortie par $r$, ce qui réduit les contraintes en courant et tension. Le bilan énergétique reste malheureusement médiocre.

Bien entendu, il existe de très nombreuses variantes de circuits d'aide, de configuration plus ou moins complexe. Dans tous les cas, on retrouve cet effet d'interaction, qui, quelle que soit la structure, en limite considérablement l'efficacité.

Il est frappant d'observer la similitude existant entre la structure à thyristors présentée plus haut (Fig. 2) et un onduleur à commutations forcées muni de réseaux d'aide (a fortiori si ces réseaux sont contrôlés). 

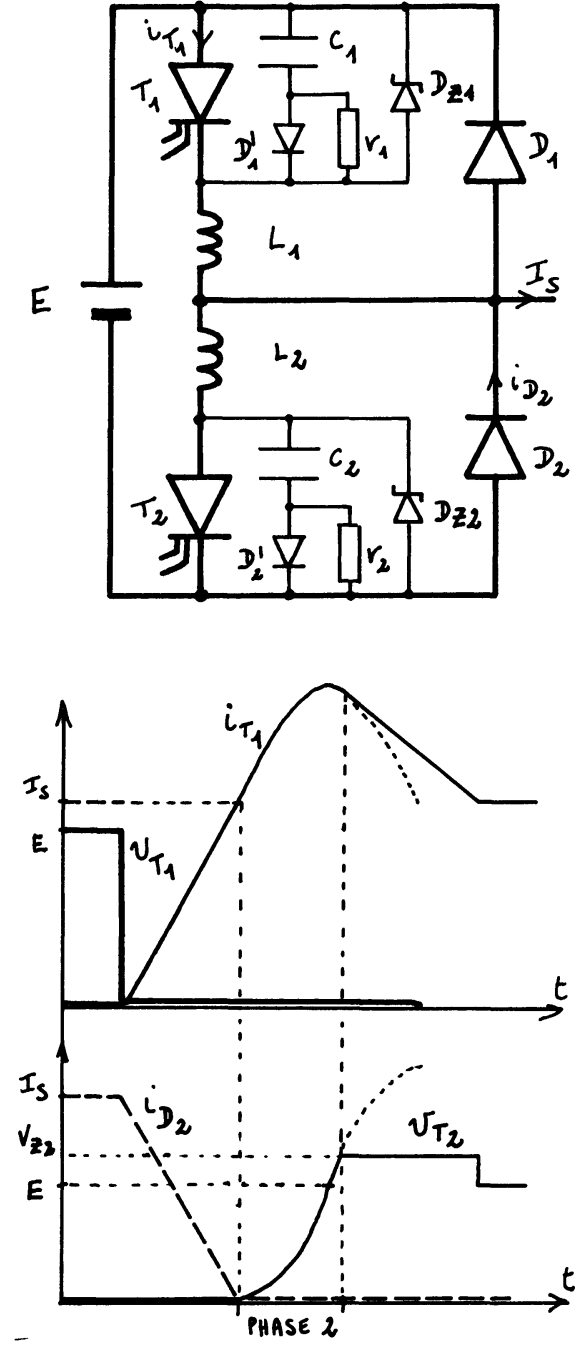

Fig. 5. - Circuits d'aide à la commutation dans un pont. [Snubber circuits in a bridge configuration.]

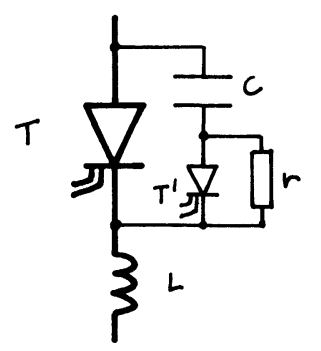

Fig. 6. - Circuits d'aide à la commutation commandés.

[Controlled snubber circuits.]

On obtient donc deux types de structures, schématiquement similaires. La structure à thyristors, qui semble inadaptée au découpage à fréquence élevée est plus saine sur le plan des commutations.

La solution qui vient alors à l'esprit est l'association des caractères les plus intéressants des deux solutions. En constituant, à l'aide d'un interrupteur entièrement commandé performant, un thyristor synthétique sans $t_{\mathrm{q}}$, il est possible de ramener la taille des circuits d'extinction à un niveau similaire à celle des circuits d'aide. Le schéma devenant quantitativement comparable, la commutation assistée lui confère un avantage indéniable.

L'autre inconvénient de ce mode, qui était la possibilité d'une perte de contrôle par non-blocage, disparaît également puisque le composant est intrinsèquement blocable. C'est une solution de ce type que nous nous proposons maintenant d'étudier.

\section{Synthèse d'un onduleur haute fréquence à commu- tation douce.}

Les principes de commutations intervenant dans les structures à thyristors sont remis au goût du jour par le développement des alimentations à découpage fonctionnant à très haute fréquence $(f>500 \mathrm{kHz})$. Différents travaux [2,3] généralisent le concept d'interrupteurs résonnants. La base de ce concept est la juxtaposition d'interrupteurs à commutation spontanée (thyristor, thyristor dual [4]) et de circuits résonnants dont les propriétés permettent de remplir les conditions de ces commutations spontanées. On obtient alors des fonctions interrupteurs globales qui, plongées dans le contexte adéquat, commutent sans perte et à gradients de courant et tension faibles en regard des fréquences de travail (commutation douce).

Lorsque l'on analyse ce concept, on note qu'il reprend et inclut l'ensemble des schémas à thyristors munis de circuits d'extinction. Paradoxalement, on est donc amené à réutiliser les principes de convertisseurs fonctionnant à basse fréquence pour franchir un pas supplémentaire quant aux performances fréquentielles.

C'est à partir de cette méthodologie que nous proposons de définir une structure d'onduleur présentant des caractéristiques intéressantes en haute fréquence.

5.1 PRINCIPES DE FONCTIONNEMENT. - Selon le concept précédemment évoqué, la structure résonante élémentaire, élaborée autour d'un thyristor, est donnée figure $7 \mathrm{a}$.

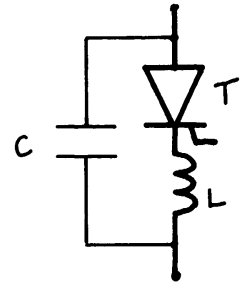

a)

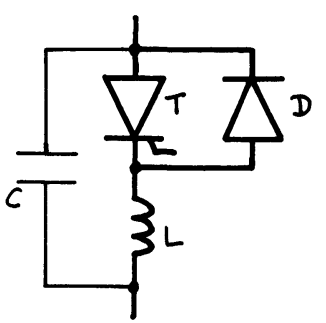

b)
Fig. 7. - Thyristors résonants.

[Resonant thyristors.] 
Une structure plus intéressante, correspondant à la nature asymétrique des composants entièrement commandés, est représentée figure $7 \mathrm{~b}$.

A partir de cette deuxième fonction, il est possible de réaliser un hacheur série (Fig. 8) dont le fonctionnement met en évidence les particularités de fonctionnement.
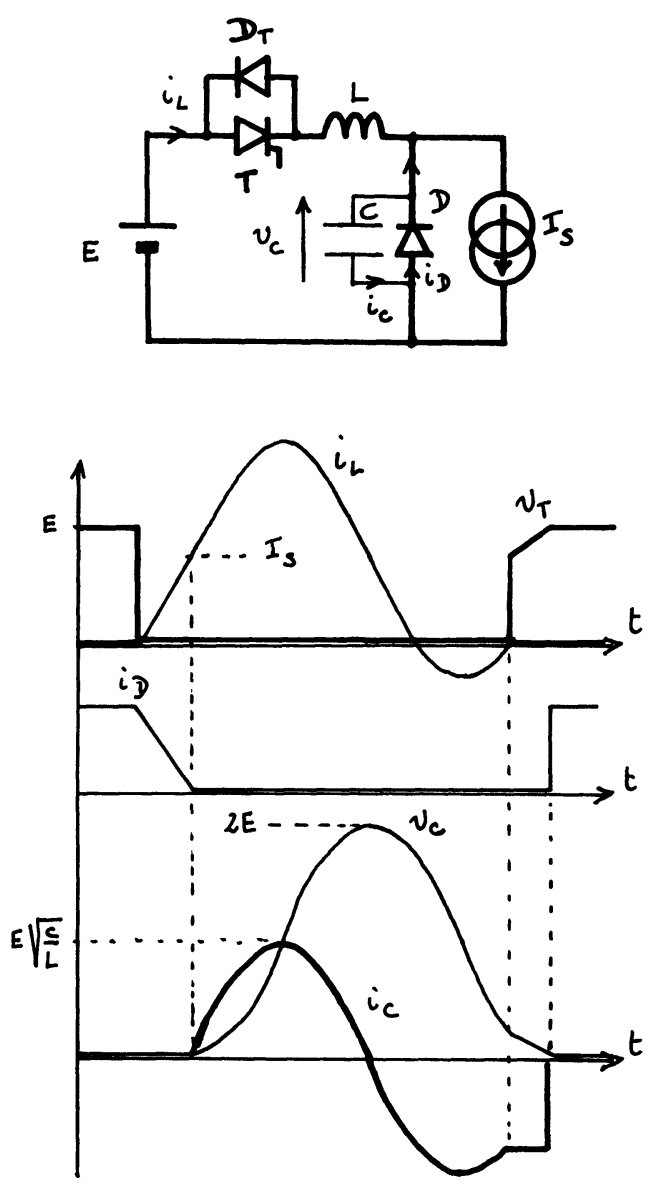

Fig. 8. - Hacheur série résonnant.

[Resonant Buck chopper.]

On retrouve, bien évidemment, un mode de commutation exempt de pertes. Une deuxième particularité est la nécessité de commander l'ensemble à fréquence variable car le temps de conduction, imposé par le circuit résonnant est sensiblement constant.

Dans une telle structure, se pose le problème de la nature des caractéristiques de sortie, $V_{\mathrm{s}}=f\left(I_{\mathrm{s}}\right)$. En régime continu de courant, ces caractéristiques normalisées sont définies par la relation :

$$
\begin{aligned}
y=\frac{V_{\mathrm{s}}}{E}=\frac{f}{2 \pi f_{0}}[ & \pi+\operatorname{Arcsin} x+\frac{x}{2}+ \\
& \left.+\left(1+\sqrt{1-x^{2}}\right)\right]=\frac{f}{2 \pi f_{0}} \cdot F(x)
\end{aligned}
$$

avec

$$
x=\frac{I_{\mathrm{s}}}{E} \sqrt{\frac{L}{C}} \quad \text { et } \quad f_{0}=\frac{1}{2 \pi \sqrt{L C}} .
$$

Lorsque l'on s'intéresse à $F(x)$, on s'aperçoit que :

$$
F(x) \simeq 2 \pi \text { pour } x<1 .
$$

En régime continu ces caractéristiques ne dépendent donc que du facteur de commande, $f / f_{0}$ (Fig. 9). Nous utiliserons par la suite les propriétés de ce convertisseur élémentaire.

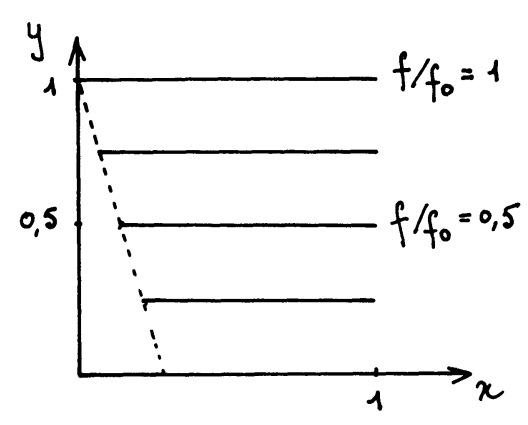

Fig. 9. - Caractéristiques de sortie.

[Output characteristics.]

Si l'on s'intéresse maintenant à la structure d'un onduleur basée sur ce principe, on peut être tenté, tout comme dans le hacheur série, de substituer, dans un bras de pont, cet interrupteur résonnant aux interrupteurs classiques. Malheureusement, on retrouve dans ce cas, entre les deux interrupteurs résonnants associés, des phénomènes d'interaction similaires à ceux rencontrés dans la constitution de réseaux d'aide à la commutation.

De la même façon, on peut tourner la difficulté, en introduisant un deuxième niveau d'interrupteurs, ce qui conduit à constituer une fonction interrupteur résonnant commandable aussi bien à l'amorçage qu'au blocage (Fig. 10a).

L'onduleur résultant, après organisation du schéma, est représenté figure $10 \mathrm{~b}$. Cette disposition a été retenue afin de permettre, dans la phase de réalisation, l'introduction d'éventuels modules, intégrant deux interrupteurs commandés. Toujours dans l'hypothèse d'un courant constant absorbé sur le bras, la séquence de commande typique et les formes d'ondes apparaissent sur les chronogrammes de la figure 11 .

L'annulation du courant dans un interrupteur principal ( $\mathrm{T} 1, \mathrm{~T} 2)$ résulte de l'amorçage d'un interrupteur auxiliaire $\left(T^{\prime} 1, T^{\prime} 2\right)$ et du régime oscillant qui suit. Le temps de conduction minimum de $\mathrm{T} 1$ et T2 doit alors être supérieur ou égal à $T_{0}$, période propre du réseau $L, C_{1,2}$. Les performances de 


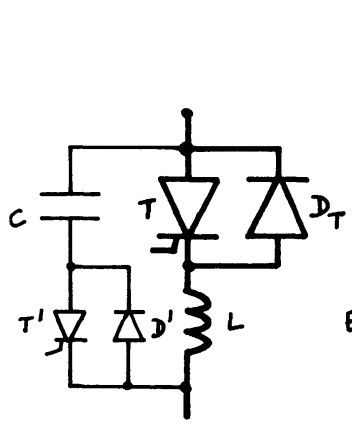

a)

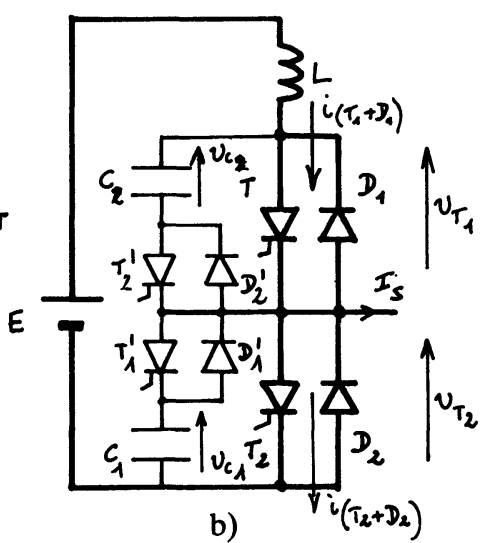

b)
- à l'observation de ces courbes théoriques, la nature des commutations est, de toute évidence, très satisfaisante ;

- le principe de fonctionnement retenu induit des contraintes instantanées de courant et tension importantes. La contrainte en tension vient de la disposition de l'inductance du réseau d'assistance, qui joue également le rôle de circuit d'aide à l'amorçage. Il peut paraître, de prime abord, absurde d'admettre un tel surdimensionnement.

Notons que les circuits d'aide, qui seraient utilisés dans une structure classique, provoqueraient sensiblement le même effet, tout en consommant une énergie considérable. D'autre part, nous verrons, dans la partie expérimentale, que la qualité des performances obtenues est à la hauteur de ces contraintes.

Ce choix peut donc parfaitement se justifier dans l'hypothèse où l'on désire réaliser un onduleur ayant des performances impossibles à obtenir avec des solutions classiques. Quel que soit le principe retenu, il semble que le surdimensionnement en contraintes

Il est important de souligner un certain nombre de points :

l'onduleur seront donc fonction de cette période propre qu'il faudra minimiser, tout en tenant compte des performances des composants utilisés.

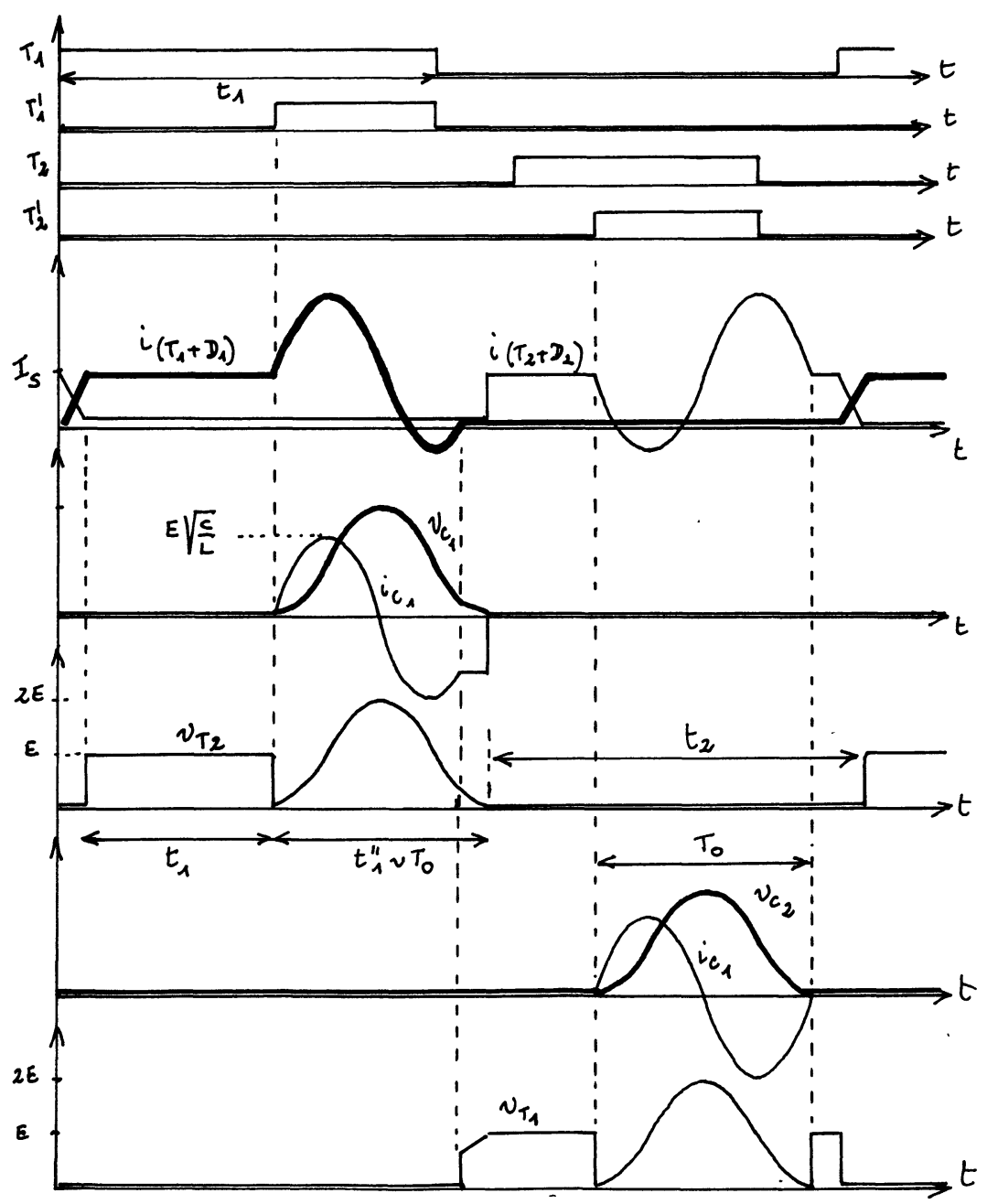

Fig. 11. - Formes d'ondes de l'onduleur résonnant.

[Resonant inverter waveforms.] 
instantanées soit un passage obligé pour l'obtention de ces performances.

Compte tenu de la forme de la tension de sortie du bras $\left(v_{\mathrm{T} 2}\right)$, il y a lieu de s'inquiéter de la dépendance de sa valeur moyenne en fonction de la grandeur de réglage, $\alpha=f \cdot t_{1}$, en supposant que l'on fonctionne, dans un premier temps, à fréquence fixe et rapport cyclique variable.

L'expression de cette valeur moyenne est :

$$
\begin{array}{cc}
V_{\mathrm{T} 2}=\left[f \cdot t_{1}^{\prime}+\frac{f}{2 \pi f_{0}}\right. & F(x)] \cdot E=f \cdot E \cdot\left(t_{1}^{\prime}+T_{0}\right) \\
\uparrow & \uparrow \\
t_{1}^{\prime} & t_{1}^{\prime \prime}
\end{array}
$$

On montre que $t_{1}^{\prime}+T_{0} \simeq t_{1}$, si bien que $V_{\mathrm{T} 2}=\alpha E$.

Le fonctionnement est donc linéaire en fonction de la grandeur de réglage.

Si l'on s'intéresse maintenant à la façon de faire fonctionner des composants entièrement commandés en mode thyristor, on remarque que la période de conduction de chacun d'entre eux est suivie de la conduction de la diode adjacente, phases qui correspondent à la mise en jeu du circuit résonnant dont on connaît la période propre. Il suffit donc de bloquer le composant concerné lorsque le courant dans la diode anti-parallèle est maximal. Cette condition est remplie à $\frac{3 T_{0}}{4}$, compté à partir de l'amorçage du composant auxiliaire correspondant. La seule règle à respecter est que la durée de conduction des diodes anti-parallèles soit suffisante pour bloquer véritablement les interrupteurs.

Dans la partie qui précède, nous avons indiqué la possibilité de fonctionner à fréquence fixe et rapport cyclique variable. Nous nous proposons, maintenant, d'introduire un mode de commande particulier permettant d'exploiter totalement les performances de l'onduleur.

5.2 MODE DE COMMANDE. - Dans la plupart des applications, la charge contient une inductance qui détermine, compte tenu de la forme de la tension délivrée par l'onduleur, l'ondulation de courant dans cette même charge. Pour déterminer cette ondulation, une approximation commode consiste à supposer, a priori, que la composante alternative haute fréquence est appliquée intégralement à cette inductance que l'on notera $L s$. Dans cette hypothèse et dans le cas d'une commande classique à $f$ constant et $\alpha$ variable, cette ondulation, $\Delta i s$, vaut :

$$
\Delta i s=\frac{E}{L s f} \cdot \alpha \cdot(1-\alpha) .
$$

Elle est maximale pour $\alpha=1 / 2$ et telle que :

$$
\Delta i s_{\max }=\frac{E}{4 L s f} .
$$

Si l'on opte pour ce mode de commande, le choix des valeurs extrêmes de $\alpha$ déterminera le rapport $f_{0} / f$. En effet, de par la nature du fonctionnement de l'onduleur, le temps de conduction minimal des interrupteurs $\mathrm{T} 1$ et $\mathrm{T} 2$ est $T_{0}$. En admettant une symétrie des valeurs extrêmes de $\alpha$, on obtient :

$$
\alpha_{\min }=1-\alpha_{\max }=\frac{T_{0}}{T}=\frac{f}{f_{0}} .
$$

Si l'on admet que les deux paramètres directeurs du dimensionnement sont $f_{0}$, fréquence propre du circuit résonnant, et $\alpha_{\min }$, valeur minimale du rapport cyclique, l'expression de l'ondulation de courant maximale devient :

$$
\Delta i s=\frac{E}{4 L s \cdot \alpha_{\min } \cdot f_{0}} \quad \text { car } \quad f=\alpha_{\min } \cdot f_{0}
$$

Pour obtenir une fréquence de découpage $f$ élevée, il faudra donc une fréquence $f_{0}$ dans le rapport $f / \alpha_{\min }$, classiquement dix fois plus élevée que $f$ si $\alpha_{\min }=0,1$. Techniquement, nous verrons qu'il est difficile de choisir une fréquence $f_{0}$ supérieure à quelques centaines de $\mathrm{kHz}$. Cela limite la valeur de $f$ à quelques dizaines de $\mathrm{kHz}$, gamme où ne sont pas exploités tous les avantages de la structure proposée.

Nous avons donc choisi un mode de commande plus approprié à cet onduleur, et qui fait intervenir une variation de la fréquence de commande.

Pour expliciter les caractéristiques de ce mode, nous nous appuierons sur les schémas de la figure 12.

La période de fonctionnement, $T^{\prime}$, résulte de l'addition des durées de conduction $t_{1}$ et $t_{2}$ des interrupteurs T1 et T2 (Fig. 12a). Ces grandeurs ont une durée minimale égale à $T_{0}$, période propre du

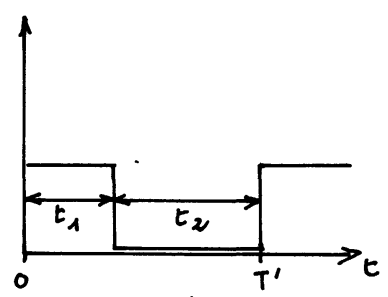

a)

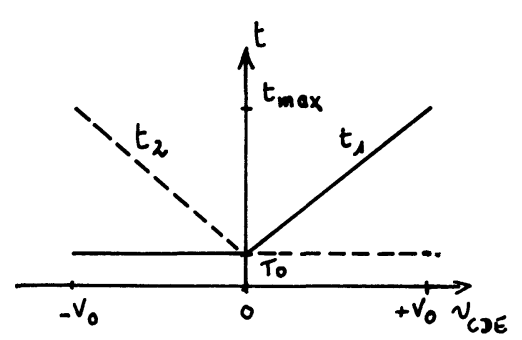

b)

Fig. 12. - Mode de commande.

[Control mode.] 
circuit résonnant. L'organe de commande réalise la fonction illustrée par la figure $12 \mathrm{~b}$. En fonction de la grandeur de commande analogique, $v_{\text {cde }}$. L'une de ces durées est figée à $T_{0}$, tandis que la seconde évolue entre $T_{0}$ et $t_{\max }$.

Si l'on travaille sur les mêmes bases que précédemment, à savoir le choix initial de $f_{0}$ et $\alpha_{\min }$, les évolutions de la fréquence de commande $f^{\prime}$, en fonction de la tension de sortie moyenne, $V_{\mathrm{T} 2}$, sont données figure 13.

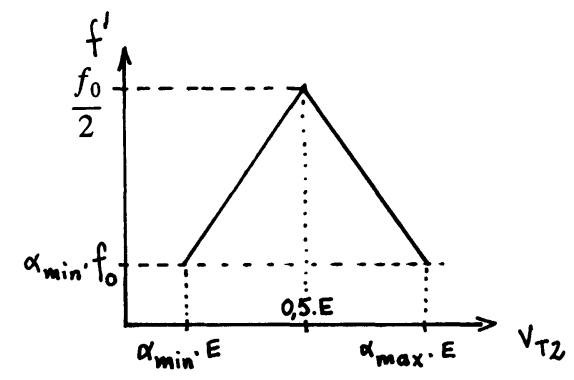

Fig. 13. - Variations de fréquence.

[Frequency variations.]

Ce mode de commande présente toutes les caractéristiques d'un contrôle à durée de conduction fixe, $T_{0}$, et fréquence variable $f^{\prime}$. L'ondulation de courant maximale, $\Delta i s_{\max }^{\prime}$, est obtenue à la fréquence la plus faible, soit ici, $\alpha_{\min } \cdot f_{0}$. Elle vaut donc:

$$
\Delta i s_{\max }^{\prime}=\frac{E}{L s \cdot \alpha_{\min } \cdot f_{0}}\left(1-\alpha_{\min }\right) \cdot \alpha_{\min } .
$$

A titre de comparaison, on peut exprimer le rapport des ondulations de courant obtenues dans les deux modes, à $\alpha_{\min }$ et $f_{0}$ identiques :

$$
\frac{\Delta i s_{\max }}{\Delta i s_{\max }^{\prime}}=4 \cdot \alpha_{\min } \cdot\left(1-\alpha_{\min }\right) .
$$

Si l'on prend l'exemple d'un rapport cyclique minimal de 0,1 , pour un même dimensionnement de l'onduleur, on obtient une réduction dans un facteur proche de trois de l'ondulation de courant. Précisons que ce type de commande est rendu possible par la nature des commutations intervenant dans la structure de puissance, qui autorise le fonctionnement à fréquence élevée.

Si les avantages de ce principe sont indéniables, la fonction correspondant à la figure $12 \mathrm{~b}$ introduit une non-linéarité de la caractéristique $V_{\mathrm{T} 2}=f\left(v_{\text {cde }}\right)$. Prenons l'exemple du quadrant où $v_{\text {cde }}>0$ :

$$
t_{2}=T_{0} \quad \text { et } \quad t_{1}=k \cdot v_{\text {cde }}
$$

d'où,

$$
V_{\mathrm{T} 2}=\frac{t_{1}}{t_{1}+T_{0}} \cdot E=\frac{k \cdot v_{\mathrm{cde}}}{k \cdot v_{\mathrm{cde}}+T_{0}} \cdot E
$$

Si l'on désire obtenir une caractéristique de réglage linéaire, il faut introduire, dans l'organe de commande, une loi du type :

$$
\frac{t_{1}}{t_{1}+T_{0}}=k \cdot v_{\mathrm{cde}} \text {. }
$$

L'organigramme général de la commande que nous avons réalisée, est donné figure 14. La grandeur de commande est un mot binaire qui peut être généré par une grandeur analogique, à travers un convertisseur analogique-numérique. Une mémoire de linéarisation permet de résoudre le problème précédemment évoquè. Les données issues de la mémoire sont utilisées pour charger les deux étages de décompteurs qui déterminent les durées $t_{1}$ et $t_{2}$. Bouclés avec deux monostables dont les impulsions correspondent aux durées de conduction minimales des interrupteurs principaux, ils constituent un oscillateur fonctionnant selon le mode de commande retenu. On notera la localisation des différents signaux nécessaires au contrôle d'un bras.

5.3 RÉAlisAtion EXPÉRIMENTALE. - Afin de valider ces principes, nous avons réalisé un onduleur monophasé résultant de la juxtaposition de deux bras (Fig. 15).

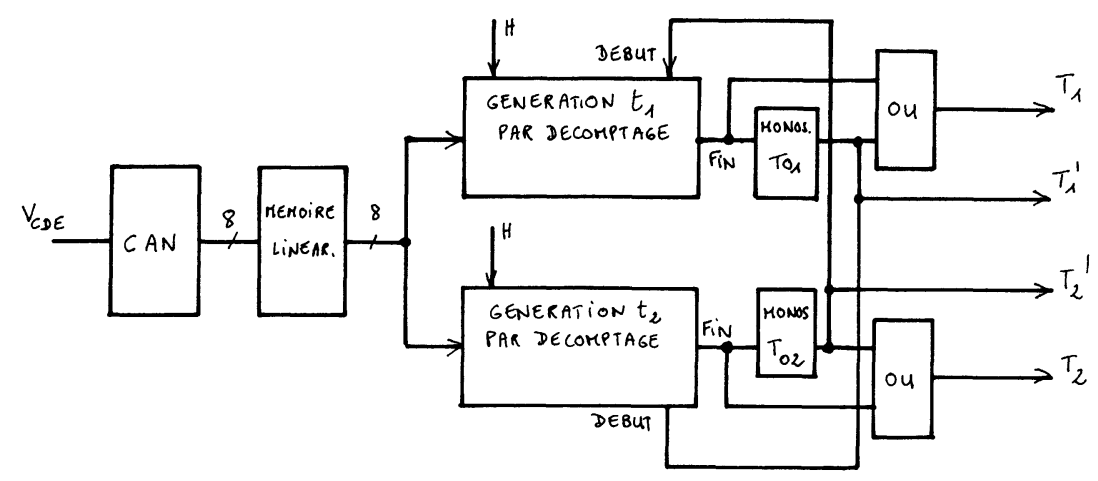

Fig. 14. - Synoptique de la commande.

[Control system.] 


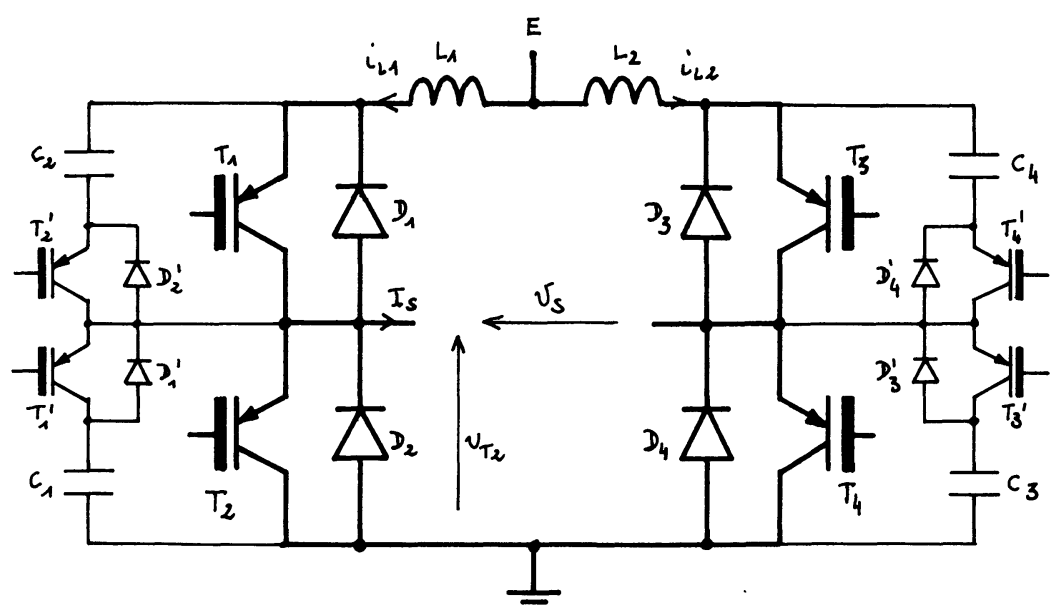

Fig. 15. - Schéma expérimental.

[Experimental scheme.]

Les composants utilisés sont des I.G.B.T. présentant des caractéristiques tout à fait adaptées à notre montage. Ces composants sont équivalents aux transistors MOS au niveau de la simplicité de la commande et des performances à la fermeture. En haute tension, ils présentent une chute de tension à l'état passant beaucoup plus faible. Inversement, le temps d'ouverture est relativement élevé. Dans notre cas, ce dernier point a peu d'importance puisque l'annulation du courant résulte de l'environnement.

La limite en courant de l'aire de sécurité en commutation étant très supérieure à la contrainte maximale en régime continu, la surintensité induite par le principe de fonctionnement de l'onduleur a peu d'influence sur le dimensionnement du composant.

Notre validation portant sur une structure alimentée en $300 \mathrm{~V}$ et capable de délivrer un courant de sortie maximale de $20 \mathrm{~A}$, les composants principaux appartiennent à la gamme $800 \mathrm{~V}-20 \mathrm{~A}$ (I.G.B.T. IXGH 20N80, diodes BYT 30PI800), tandis que les composants auxiliaires sont du type $500 \mathrm{~V}-10 \mathrm{~A}$ (I.G.B.T. RCP $10 \mathrm{~N} 50 \mathrm{~A}$, diodes BYT 12PI800).

Le choix de la fréquence propre du circuit résonnant est essentiellement fonction des performances des diodes rapides qui interviennent dans la dernière phase de l'oscillation et dans les configurations de roue libre. Pour obtenir des performances satisfaisantes, le choix d'une fréquence $f_{0}$ de $250 \mathrm{kHz}$ semble, après évaluation préliminaire, raisonnable. Cette valeur et la contrainte sur le pouvoir de coupure $\left(I_{s}=20 \mathrm{~A}\right)$, déterminent entièrement le couple $L, C$ :

$$
\begin{aligned}
& L=10 \mu \mathrm{H} \\
& C=66 \mathrm{nF} .
\end{aligned}
$$

En choisissant $\alpha_{\min }=0,1$, la fréquence de fonctionnement théorique évolue entre 25 et $125 \mathrm{kHz}$. Intro- duits dans la commande pour des raisons de sécurité, différents temps morts font que la fréquence effective est comprise entre 20 et $100 \mathrm{kHz}$. L'onduleur étant commandé en mode bipolaire, les signaux de contrôle destinés à T1-T4, $T^{\prime} 1-T^{\prime} 4, T 2-T 3, T^{\prime} 2-T^{\prime} 3$, sont identiques.

Lorsque l'on observe les signaux présentés figure 11 , on remarque que pour un courant de sortie d'un signe donné, l'une des deux oscillations apparaissant sur une période de fonctionnement est inutile. Les contraintes supplémentaires qu'elle induit sur les différents composants sont importantes. Nous avons donc introduit une fonction supplementaire dans la commande, en détectant le signe du courant délivré par l'onduleur. En fonction de ce signe, l'un ou l'autre signal de commande des interrupteurs auxiliaires est inhibé.

De surcroît, ces signaux sont simultanément inhibés dans la gamme $-0,5 \mathrm{~A}<I_{s}<0,5 \mathrm{~A}$. En effet, les problèmes de commutation sont quasi inexistants à faible courant. On profite donc de l'utilisation d'interrupteurs intrinsèquement blocables pour minimiser les contraintes imposées par cette structure.

L'introduction de cette option est surtout motivée par la difficulté de réaliser l'inductance $L$ si l'on conserve le fonctionnement intrinsèque de l'onduleur. Les utilisateurs des principes de la résonance à haute fréquence savent que ces principes mènent généralement à un transfert de contraintes, des dispositifs à semi-conducteurs vers les composants passifs des circuits résonnants. Notre structure n'échappe pas à cette règle et le fait de diviser par deux la fréquence de répétition des oscillations de blocage facilite grandement le dimensionnement de l'inductance $L$.

Le fonctionnement obtenu est alors très satisfaisant. La qualité des formes d'ondes obtenues en mode continu-continu (Figs. 16a, b, c) et en mode continu alternatif (Fig. 16d), sur charge $L s, R s$, sans 


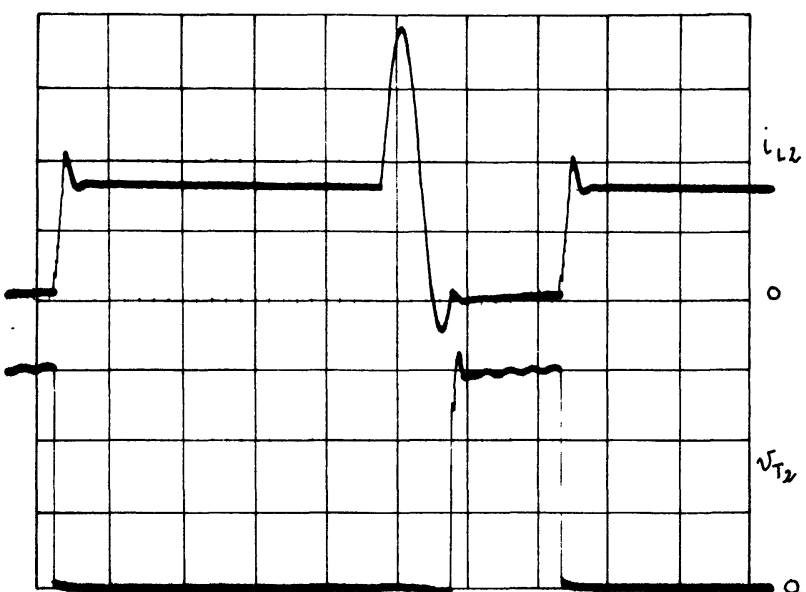

a)

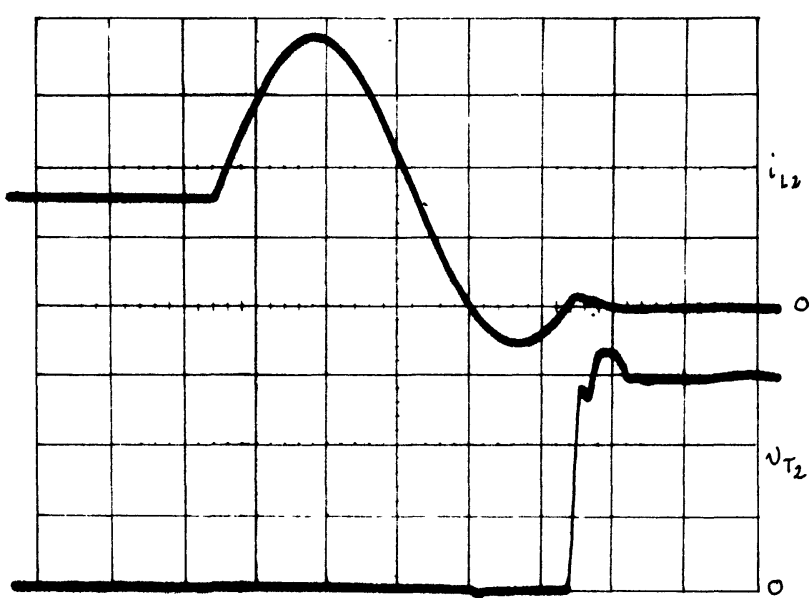

c)

Fig. 16. - Résultats expérimentaux.

(a) Mode Continu-Continu ; $I_{\mathrm{S}}<0 ; i_{\mathrm{L} 2}: 10 \mathrm{~A} / \mathrm{C} ; v_{\mathrm{T} 2}$ : $100 \mathrm{~V} / \mathrm{C} ; t: 5 \mu \mathrm{s} / \mathrm{C}$.

(b) Mode Continu-Continu ; $I_{\mathrm{S}}>0 ; i_{\mathrm{L} 1}: 10 \mathrm{~A} / \mathrm{C} ; v_{\mathrm{T} 2}$ : $200 \mathrm{~V} / \mathrm{C} ; t: 5 \mu \mathrm{s} / \mathrm{C}$.

problèmes particuliers de mise en œuvre, constitue un des avantages de ce procédé.

Les rendements obtenus, pour une puissance de sortie de $4 \mathrm{~kW}$ en mode continu-continu, de $2 \mathrm{~kW}$ en mode continu-alternatif, sont respectivement de 96 et $98 \%$. Associés à la valeur importante des fréquences de fonctionnement, ces différents éléments, dans des applications où les performances sont primordiales, semblent largement justifier la relative complexité du schéma et les contraintes supplémentaires qui en découlent.

Les relevés de la figure 17 confirment l'intérêt du mode de commande utilisé. Sur ces relevés apparaissent les contenus harmoniques des tensions de sortie obtenues, d'une part dans notre structure (Fig. 17a), d'autre part dans un onduleur classique à transistors MOS (Fig. 17b) présentant les mêmes caractéristi-

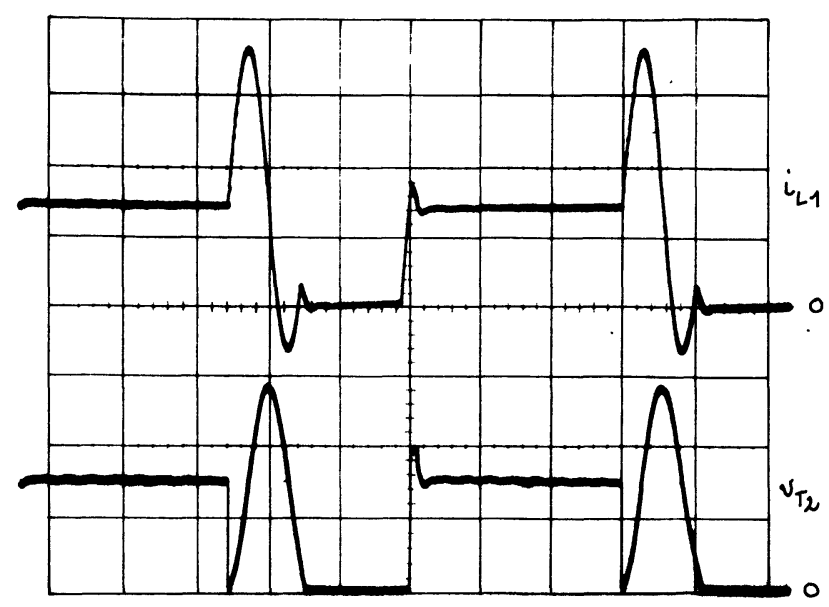

b)

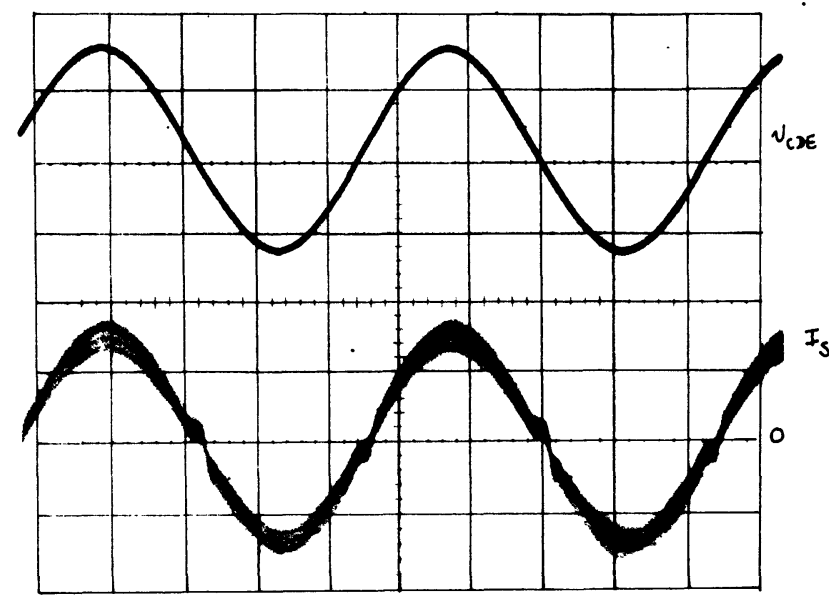

d)

(c) Mode Continu-Continu, Détail de la commutation ; $I_{\mathrm{S}}<0 ; i_{\mathrm{L} 2}: 10 \mathrm{~A} / \mathrm{C} ; v_{\mathrm{T} 2}: 100 \mathrm{~V} / \mathrm{C} ; t: 1 \mu \mathrm{s} / \mathrm{C}$.

(d) Mode Continu-Alternatif ; $L_{\mathrm{S}}<500 \mu \mathrm{H} ; \quad I_{\mathrm{S}}$ : $10 \mathrm{~A} / \mathrm{C} ; t: 1 \mathrm{~ms} / \mathrm{C}$.

[Experimental results.]

ques électriques mais commandé classiquement à fréquence fixe $(20 \mathrm{kHz})$ et rapport cyclique variable. Ces tensions correspondent à une modulation sinusoïdale à $200 \mathrm{~Hz}$ du signal haute fréquence. Les rapports cycliques extrêmes équivalents ainsi que l'amplitude de la composante fondamentale de $v_{\mathrm{s}}$ ainsi obtenue, sont identiques dans les deux cas.

Dans la bande des fréquences de découpage, le filtrage des harmoniques correspondantes apparaît beaucoup plus aisé dans le premier cas. Dans la bande basse fréquence, quelques harmoniques apparaissent dans notre onduleur. Elles sont dues à une non-linéarité de la commande (bande morte) dans la zone des fréquences maximales de travail, donc au passage par zéro. Ce problème peut aisément être résolu en sophistiquant l'étage de commande, tout en conservant le même principe de contrôle. 


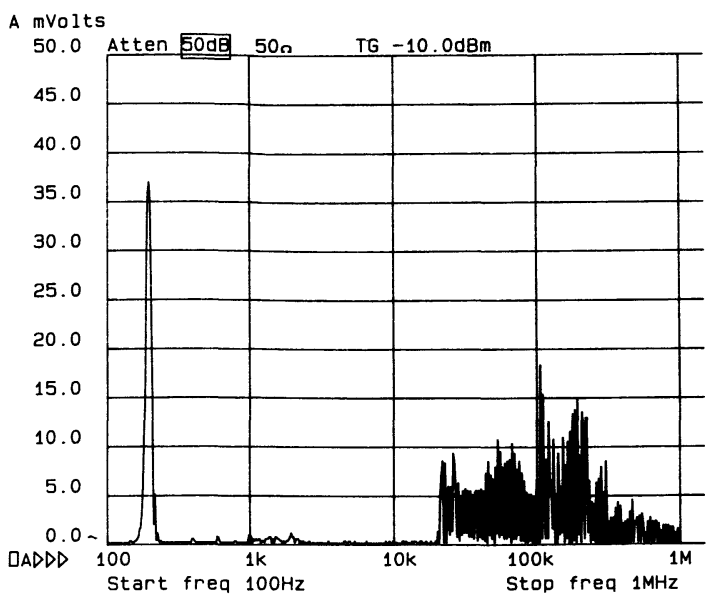

(a) Onduleur à commutation douce.

Fig. 17. - Comparaison spectrale.

[Spectrums comparison.]

\section{Conclusion.}

L'observation des schémas, utilisés dans la constitution d'onduleurs de tension destinés à la commande en modulation de largeur d'impulsion, montre que la présence de circuits annexes est un mal nécessaire lorsque l'on veut effectuer un découpage à fréquence élevée. En attribuant, à ces circuits, une fonction qui apporte une solution satisfaisante aux problèmes cruciaux posés par les commutations, sans qu'ils soient le siège de pertes autres que celles dues aux imperfections des composants, on justifie amplement les contraintes instantanées supplémentaires que subissent les composants principaux. Ces contraintes ne constituent pas un handicap insurmontable dans

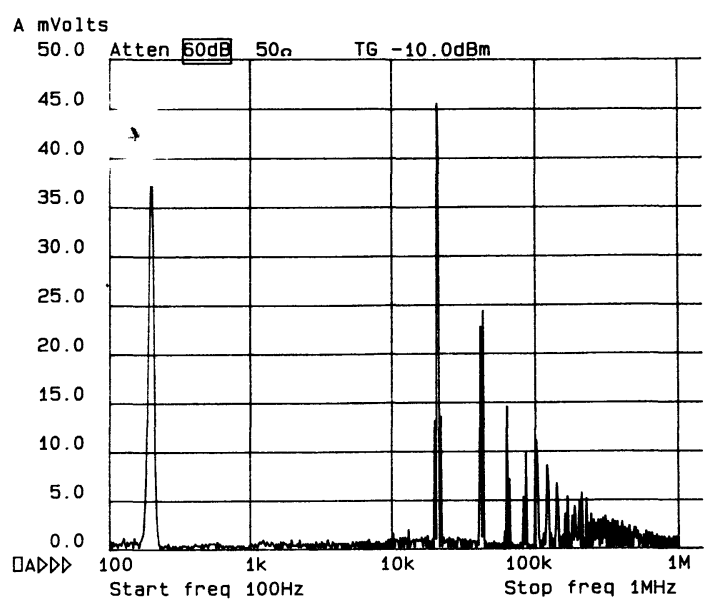

(b) Onduleur classique.

la mesure où le principe autorise la mise en série de composants sans contrôle complexe (contrainte de tension) et un choix judicieux du type de composant minimise l'effet des surintensités de blocage.

Dans l'exemple présenté, les puissances mises en jeu sont faibles tandis que les fréquences de fonctionnement sont importantes. On peut notablement déséquilibrer ce rapport afin de privilégier la puissance en décalant vers le bas la gamme des fréquences de travail. Associé au mode de commande proposé ici, ce principe devrait permettre d'améliorer très sensiblement, par rapport aux solutions traditionnelles, les performances des onduleurs de tension destinés à la commande de machines ou à l'élaboration d'alimentations de secours.

\section{Bibliographie}

[1] Bergmann C., Contribution à l'étude de la commande numérique directe de moteurs synchrones à aimant-modélisation, réalisation, expérimentations, thèse de doctorat de l'université Paris VI (5 mars 1986).

[2] Kwang-Hwa Liu, C. Lee F., Resonant switches. A unified approach to improve performances of switching converters, IEEE international telecommunications energy conference (1984) pp. 344-351.

[3] Meynard T. A., Cheron Y., Foch H., Generalization of the resonant switch concept structures and performances, Second european conference of power electronics and applications 1 (1987) pp. 239-244.
[4] Cheron Y., Application des règles de la dualité à la conception de nouveaux convertisseurs à transistors de puissance. Synthèse du thyristor dual. Domaine d'application, Thèse de docteur-ingénieur, Toulouse (1982).

[5] Pearson W. R., Sen P. C., Designing optimum snubber circuits for the transistor bridge configuration, Electric machines and power systems, 8 (1983) pp. 321-332.

[6] Ferrieux J. P., Forest F., Alimentations à découpage, Convertisseurs à résonance (Edition Masson) 1987.

[7] Jayant-Baliga B., Chang M., Shafer P., SMith M. W., The insulated gate transistor-a new power device, IEEE ind. appl. soc. meet. (1983) pp. 794-803. 